\title{
A framework for curriculum design to support mathematical sense making
}

\author{
Julian D. Gifford and Noah D. Finkelstein \\ Department of Physics, University of Colorado Boulder, Box 390, 80309
}

An approach to the design of curriculum supporting student engagement in mathematical sense making (MSM) is discussed. This approach draws from a categorical framework for MSM that is situated in the PER and science education literature on mathematical modeling and sense making. Two pieces of curricula spanning both content (the photoelectric effect and geometric optics) and course context (STEM-major versus non-STEM-major) designed using this framework are analyzed for the reasoning structures they are designed to scaffold.

2020 PERC Proceedings edited by Wolf, Bennett, and Frank; Peer-reviewed, doi.org/10.1119/perc.2020.pr.Gifford

Published by the American Association of Physics Teachers under a Creative Commons Attribution 4.0 license.

Further distribution must maintain the cover page and attribution to the article's authors. 


\section{INTRODUCTION}

As part of a larger project aimed at understanding and supporting mathematical sense making (MSM), we have developed a framework for understanding student reasoning that uses both mathematical and physical tools $[1,2]$. This framework builds on existing work on mathematical modeling [36], is situated in the sense making literature [7], and has a theoretical (cognitive) basis in mediated cognition [8] and broader socio-cultural theories of learning [9-11].

To date, this work has primarily been employed to analyze student reasoning [1, 2]. Here, we present a preliminary application of this framework to curriculum design. This present work is not (yet) seeking to examine impact on student reasoning; rather it is a demonstration of how curricula might be designed based on this framework. The effects of these curricula will be the subject of future work. This paper will begin with a brief summary of the framework and how it leads to guiding principles for curriculum design, then two pieces of curriculum that have been designed to promote MSM based on this approach will be analyzed.

\section{THE MSM FRAMEWORK}

The basic units of the framework are four sense making modes, which draw from Vygotsky's theory of mediated cognition [8]. The four modes are defined by the characteristics of the object and the tool of sense making (specifically whether they are mathematical or physical in nature). As an example: a plot is a graphical representation showing a functional relationship between two quantities, and so is a fundamentally mathematical entity with aspects like slope, concavity, asymptotes, discontinuities etc. However, the plots drawn in physics show the relationship between physical quantities, not simply abstract variables, and so carry information about phenomenon in the world.

Consider a plot of the maximum kinetic energy of ejected electrons in a photoelectric effect experiment versus the frequency of incident light, shown in solid blue in Fig. 1. There is a discontinuity in the slope of this plot; arguing that this discontinuity suggests a change in the functional dependence of the equation for $K E(f)$ is an example of Msm-M reasoning ("Mathematical sense making of a mathematical object"), where the plot is used as a (mathematical) tool to reason about the (mathematical) function it represents. However, arguing that this discontinuity suggests a fundamental change in the interaction between light and the metal plate - that the photon energy is insufficient to free an electron from the metal - is an example of Msm-P reasoning ("Mathematical sense making of a physical object"), where the plot is used as a (mathematical) tool to understand the (physical) behavior of electrons in the experiment. Alternatively, generating this plot could involve Psm-M reasoning ("Physical sense making of a mathematical object") employing a physical model as a tool to argue that since kinetic energy cannot be negative the plot

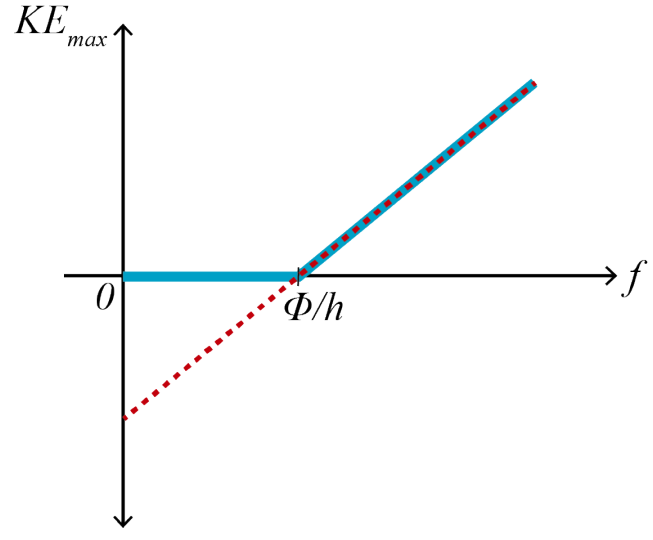

FIG. 1. (Color) Plots of the equation $\mathrm{KE}_{\max }=h f-\Phi$ (dashed red) and the maximum kinetic energy of ejected electrons versus the frequency of light in a Photoelectric Effect experiment (solid blue).

of $K E(f)$ (a mathematical object) cannot include a portion below zero. Psm-P reasoning ("Physical sense making of a physical object") would not directly involve the plot, a mathematical entity, but could be used to argue that since the kinetic energy of the electron comes from the incident photons, higher frequency light should lead to a greater $\mathrm{KE}_{\max }$. Representations of the four modes of the framework are shown in the left of Fig. 2.

While the use of one of these modes in isolation can constitute robust sense making, the larger activity of sense making is often involves shifts between and combinations of these modes. Three processes are particularly important: translation is a general shift in reasoning structure in which either the object or tool (or both) change from a prior mode without an explicit connection to prior reasoning; chaining is a specific translation, where the object of the prior reasoning mode becomes the tool of the future mode; and coordination is the combination of two different modes that use different tools to understand the same object. An abstract representation of these processes is shown in the right of Fig. 2.

The outcome of these three processes is a complicated reasoning structure, also shown in Fig. 2. If the individual modes are considered to be atoms of reasoning, this more complicated reasoning structure is a molecule of sense making. Several different molecules of sense making have been observed in prior and ongoing work that spans both course content and student populations in physics. This framework's focus on tool use to scaffold reasoning is aligned with a resources perspective [12], where cognition is viewed as the activation of multiple resources rather than as the application of a single, well-developed conception (or misconception) [13].

From this framework we distill several guiding principles for the design of curriculum. First, that robust reasoning about entities often involves the coordinated use of multiple reasoning modes and multiple tools. Thus, the generation of coordinated reasoning structures (like the one shown in Fig. 2) that can be applied flexibly in future situations 


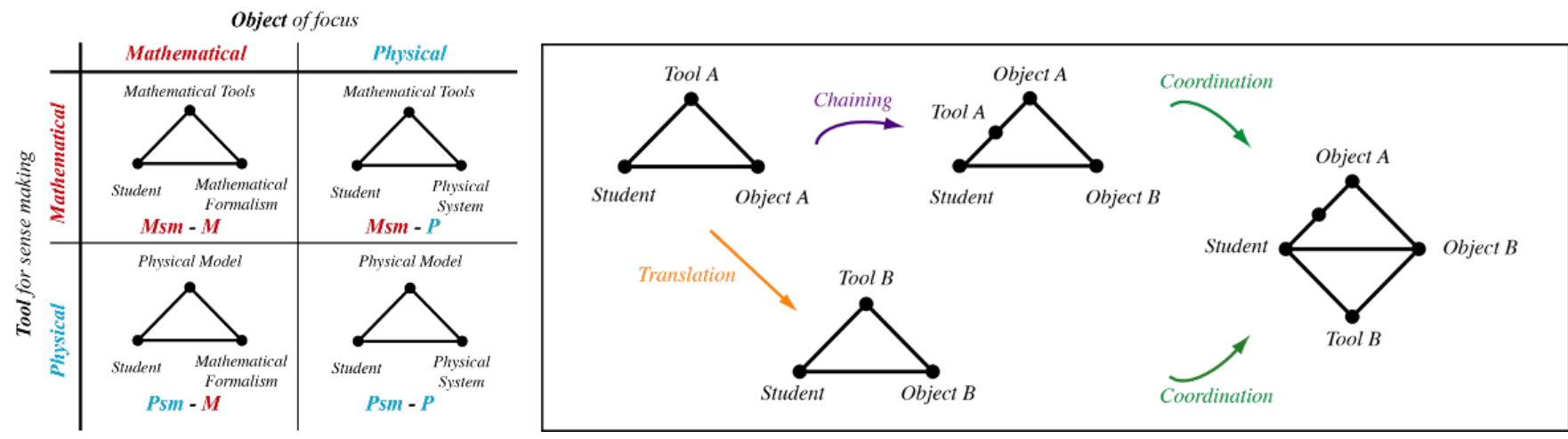

FIG. 2. LEFT: The four basic modes of the sense making framework, which differ in the tool and object of sense making. RIGHT: A sense making diagram, showing representations of the processes of translation, chaining, and coordination. The node (dot) following chaining represents the prior use of Tool A to understand Object A, and the square structure at the end represents the coordinated use of two tools.

are an end goal of instruction. This principle aligns with higher order mediated cognition $[8,10]$. Second, the proficient use of a particular tool (either in isolation or in coordination with others) generally requires knowledge about that tool, which can arise from chaining. The repeated use of tools, and the chaining of tools to understand varied and more complicated objects, is a fundamental step in developing more complicated reasoning structures. Knowledge of tool use aligns with meta-representational competence as a goal of instruction [14]. Finally, the activation of coordinated modes of reasoning and of meta-representational competencies are metacognitive skills of their own. While this activation should initially be highly targeted during instruction, this scaffolding should decrease in extent throughout instruction in line with the scaffolding and fading technique [15].

Preliminary results from our studies of student reasoning suggest that the targeted activation of particular tool use does scaffold student reasoning and answer making. The framework could be employed to give a measure of how frequently various curricula give students opportunities to engage in different modes of reasoning or to combine these modes. While this is a topic of future work, the present work is an introduction to how curricula might be designed based on this framework.

\section{ACTIVITY I: THE PHOTOELECTRIC EFFECT}

Much prior work has been done to study student difficulties with the photoelectric effect $[16,17]$ and with the hidden mathematical structure of the expression for the maximum kinetic energy of ejected electrons $\left(\mathrm{KE}_{\max }=h f-\Phi\right)$ [18]. In part to address these existing difficulties, and to demonstrate the application of the MSM framework to curriculum design, we have developed and implemented a tutorial that helps students build up the photon model of light based on the experimental results of the Photoelectric Effect. The major focus of the present analysis will be on a portion of this tutorial that supports students in developing coordinated MSM reasoning structures around the kinetic energy of ejected electrons. In particular, the (incomplete) equation $\mathrm{KE}_{\max }=h f-\Phi$ (the dashed red plot in Fig. 1) and its alignment with experimental data (the solid blue plot in Fig. 1) and the photon model of light. A version of this tutorial is available online [19].

This tutorial begins with an exploration of the Photoelectric Effect Experiment using the PhET simulation [20]. In this section, students explore the parameters of the experiment (intensity and frequency of light, battery voltage, material of the metal plate, measured current), and are asked how these quantities could be used to measure the rate of electron ejection (equal to the current at positive battery voltage) and the maximum kinetic energy of the ejected electrons (related to the "stopping potential" - the battery voltage required to bring the current to zero $-\mathrm{KE}_{\max }=e V_{\text {stop }}$ ). These questions are designed to engage students in a Psm-P mode of reasoning, where the PhET sim is used as a proxy for the physical experiment to develop an experimental (if not mechanistic) understanding of the phenomenon.

Students are then asked to predict the outcome of two experiments using a wave model for light, after which the tutorial turns to experimental results. Students are presented with a plot of the measured current versus the battery voltage (an $I V$ plot) for a particular experiment and are asked to consider (1) the experimental setup that led to this plot (Psm-M - the physical meaning of a mathematical object) and (2) what the plot does and does not show about the behavior of the ejected electrons in this experiment (chained Msm-P). The bulk of this section is devoted to developing a physical model for light, based on plots generated from an experiment. As the plot is a fundamentally mathematical entity this is an Msm-P mode of reasoning; however, understanding the plot requires an understanding of the physical experiment (Psm-P), so this is an instance of chained Msm-P. Through explicit chaining of tool use, the curriculum is designed to scaffold students into generating the reasoning structure shown in Fig. 3.

To support the development of the photon model, the tutorial shifts to consider the (maximum) kinetic energy of the ejected electrons. The reasoning structure scaffolded by this 


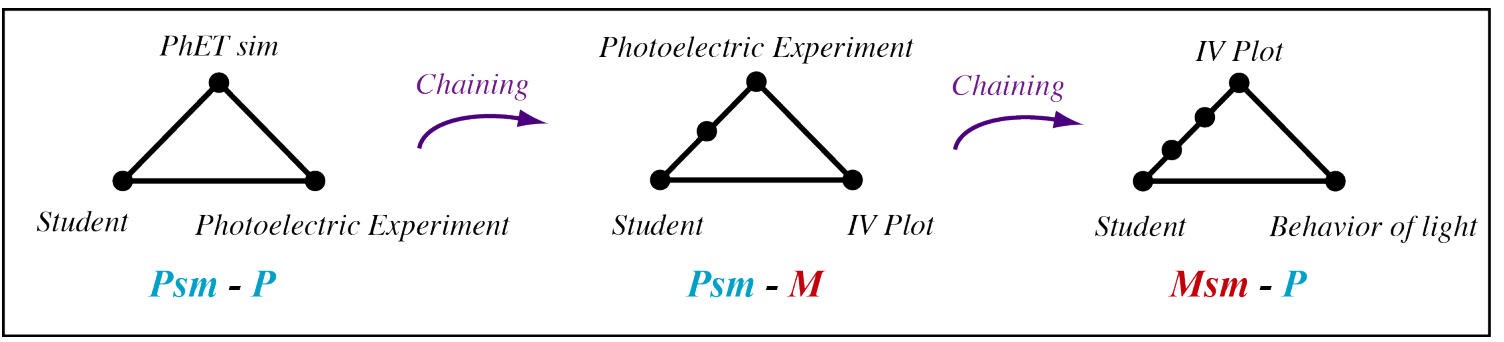

FIG. 3. A sense making diagram showing the reasoning structure developed in the first half of the tutorial. Through repeated chaining, experimental plots of current versus battery voltage are ultimately used to understand the behavior of light and the failure of the wave model.

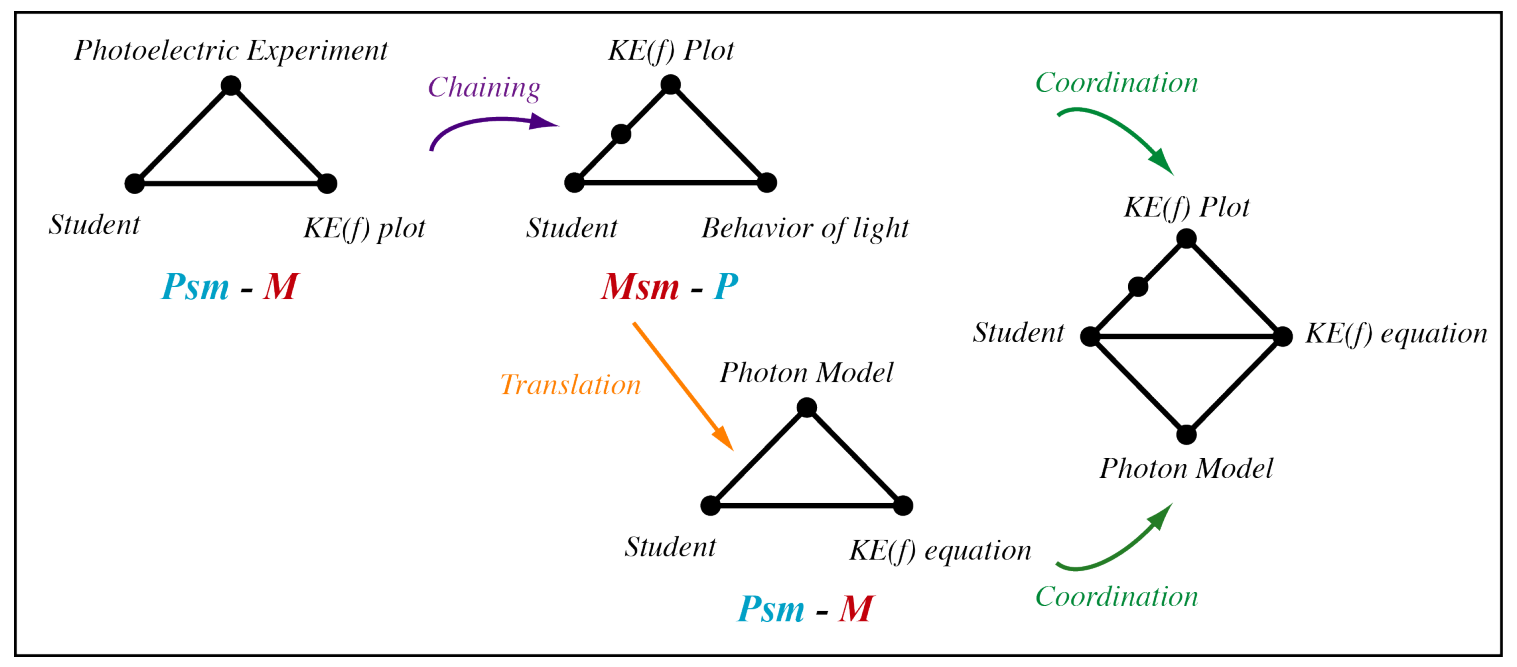

FIG. 4. A sense making diagram showing the coordinated reasoning structure developed to understand the expression $K E(f)=h f-\Phi$ using both mathematical (the experimental plot of $K E(f)$ ) and physical (the photon model of light) tools.

tutorial is shown in the sense making diagram in Fig. 4, and aims to help students make sense of the standard (incomplete) equation for $K E_{\max }$ using both mathematical (the experimental plot) and physical (the photon model) tools. First, students are asked to consider the physical experiment that must be conducted to generate the plot of kinetic energy versus frequency (Psm-M); they then conduct the experiment in PhET for two different metal plates and interpret the physical significance of these plots, comparing these interpretations with the predictions of the wave model of light (chained Msm-P).

Following an introduction to the photon model, students are asked to develop an equation modeling the kinetic energy of the ejected electrons in terms of the incident light - this shift is a translation in reasoning structure. They are explicitly told to use energy considerations, and so a physical model (the photon model) is used as a tool to develop a mathematical expression (Psm-M). Students are then asked for the range of validity of this expression and are told to consider the plots of $K E(f)$ from earlier. These plots show an explicit "cutoff frequency" below which no electrons are ejected. From their plots, students can see that changing the material of the plate (the work function) alters this cutoff frequency but does not alter the slope of the graph above this value. By coordinating their understanding of the plot with their understanding of the equation, students determine that the expression is only valid when the energy of the light is equal to or greater than the work function of the material $(h f \geq \Phi)$.

The ultimate end goal of the tutorial is the coordination of the IV plots and plot/equation of $K E(f)$ as tools to understand the overall physical behavior of light (modeled as a photon) and the ejected electrons. Anecdotally, based on our interactions with students as they work through this tutorial and in our analyses of student reasoning in an end of semester focus group $[1,2]$, this approach is promising, though a more robust measurement of its efficacy is a topic of future work.

\section{ACTIVITY II: THE LENS EQUATION}

The second piece of curriculum comes from a different course context: a general education physics course intended for non-science-majors that focuses on the behavior of light and human perception of color. As the population was notably different from a modern physics context, in particular with their general comfort level with mathematics, an activity was designed to bridge the gap between the conceptual rea- 


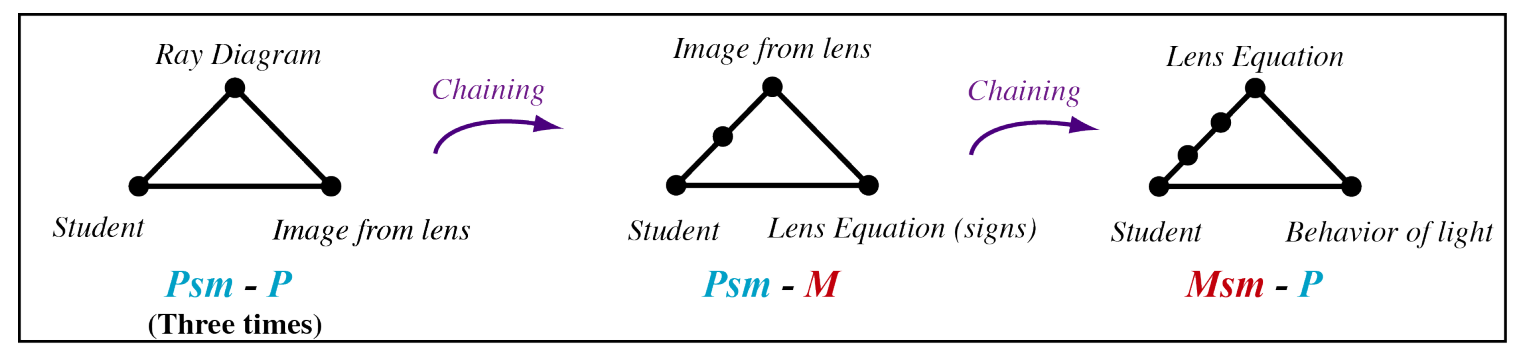

FIG. 5. A sense making diagram showing the multiply chained reasoning structure developed in the Lens Equation Activity.

soning (physical) and mathematical approaches to lens systems. This activity, the second in a set of two in class activities, is focused on diverging lenses and the lens equation and is available for review online [21].

The activity begins with a simple ray diagram for an extended object (a pencil) near a diverging lens. This begins the process of using ray tracing as a (physical) tool to understand the physical behavior of the system (where does the image form, is the image real or virtual). After the first diagram, students are asked how they should move the pencil (towards or away from the lens) to make the image larger, and then asks to explain how (if at all) they could move the pencil such that the image is larger than the object - note that this is impossible with a diverging lens. These questions aim to develop comfort with the process of drawing the principle rays for the given lens, scaffolding comfort with ray diagrams as a tool for understanding the behavior of light. All three of these questions encourage Psm-P reasoning.

Once comfort with using ray tracing as a tool to understand the physical system is developed, students are then provided with an explanation of the sign conventions for the lens equation. Using a converging lens (from the previous activity) as an example, students are told how to assign signs to each of the relevant terms in the lens equation (the image distance, image height, and focal length) based on the characteristics of the image in their ray diagrams. This chained Psm-M mode of reasoning, where the image from the ray diagram is used as a tool to understand the signs of the lens equation, is the center reasoning structure in Fig. 5. Finally, once the signs of the lens equation have been given physical significance, the lens equation is used as a formal mathematical tool to determine the type of lens given the magnitude of the image and object distances, and the type of image (virtual). This involves a second round of chaining, as shown in Fig. 5.

Preliminary indications show that students do follow this reasoning pathway when working through this activity; however, there is limited student data as this activity was implemented immediately following a transition to emergency remote teaching in response to the COVID-19 pandemic. Deeper investigations of the outcomes of this activity, as well as any iterative modifications, are the topic of future work.

\section{v. CONCLUSIONS}

This paper has presented an approach to curriculum design that promotes mathematical sense making. In particular, scaf- folding engagement in the four basic modes (Msm-M, Msm$\mathrm{P}$, Psm-M, and Psm-P) and the generation of more complicated reasoning structures through the processes of translation, chaining, and coordination.

Two pieces of curricula designed using this approach have been analyzed for the reasoning structures they scaffold. These curricula span both content (modern physics and geometric optics) and course context (STEM and non-STEM majors). The photoelectric effect tutorial was designed in parallel with an extensive analysis of student reasoning regarding the photoelectric effect and mathematical sense making, and drew from previous work on conceptual and mathematical student difficulties [16-18].

We argue that it is possible to design curriculum based on this framework both by drawing from research in student reasoning and also a priori, without an explicit study of student difficulties. For example, the Lens Equation activity did not draw explicitly on documented difficulties, rather it scaffolded a specific reasoning structure that the instructors believed would prove productive. While the second approach to designing curriculum using this framework does not rely specifically on documented difficulties, it is based in theories of cognition and does rely on the understanding of content experts as well as the cultural and historic use of various tools.

Though we do argue for the importance of engaging students in multiple modes and scaffolding coordinated reasoning structures, we make no claims regarding the relative importance of the individual modes. Instead, the framework offers a flexible structure based on the goals of the instructor. In particular, the framework could be used to establish a measure of how frequently various instructional materials provide opportunities for engagement in each of the modes, as well as scaffold the intersection and coordination of these modes. This measure can provide an instructors a sense of how well the curricula align with their established learning goals. Testing the efficacy of the curricula presented here is the subject of ongoing and future work.

The authors would like to thank the PER group at CU Boulder for insightful discussions and feedback on the framework and curriculum. This work was supported by NSF: 1625824 . 
[1] J. D. Gifford and N. D. Finkelstein, Categorizing mathematical sense making and an example of how physics understanding can support mathematical understanding, presented at the Physics Education Research Conference 2019, Provo, UT (2019).

[2] J. D. Gifford and N. D. Finkelstein, A categorical framework for mathematical sense making in physics. Manuscript submitted for publication (2020).

[3] B. R. Wilcox, et al., Analytic framework for students' use of mathematics in upper-division physics, Phys. Rev. ST Phys. Educ. Res. 9, 020119 (2013).

[4] T. J. Bing and E. F. Redish, Analyzing problem solving using math in physics: Epistemological framing via warrants, Phys. Rev. ST Phys. Educ. Res. 5, 020108 (2009).

[5] O. Uhden, et al., Modelling Mathematical Reasoning in Physics Education, Sci. \& Educ. 21(4), (2012).

[6] E. Kuo et al., How students blend formal and conceptual mathematical reasoning in solving physics problems, Science Education, 97: 32-57 (2013).

[7] T. O. B. Odden and R. S. Russ, Defining sensemaking: Bringing clarity to a fragmented theoretical construct, Science Education, 1-19 (2018)

[8] L. Vygotsky, Mind in Society (Harvard University Press, 1978).

[9] Y. Engestrom, R. Miettinen, and R. Punamaki, eds., Perspectives on activity theory (Cambridge University Press, 1999)

[10] M. Cole, Cultural Psychology: A Once and Future Discipline
(Harvard University Press, 1998)

[11] W. M. Roth, Cultural-historical activity theory: Vygotsky's forgotten and suppressed legacy and its implication for mathematics education, Math Ed. Res. J. 24, 87 (2012).

[12] D. Hammer, Student Resources for Learning Introductory Physics, Am. J. Phys. 68, S45 (2000)

[13] A.A. DiSessa and B. L. Sherin, What changes in conceptual change?, International Journal of Science Education, 20:10, 1155-1191 (1998)

[14] A. A. DiSessa, Metarepresentation: Native competence and targets for instruction, Cognition and instruction, 22(3), 293331 (2004)

[15] B. Rosenshine and C Meister, The Use of Scaffolds for Teaching Higher-Order Cognitive Strategies, Educational Leadership, 49 (1992)

[16] R. N. Steinberg, G. E. Oberem, and L. C. McDermott, Development of a computer-based tutorial on the photoelectric effect, Am. J. Phys, 64, 1370 (1996)

[17] S. B. McKagan, W. Handley, K. K. Perkins, and C. E. Wieman A research-based curriculum for teaching the photoelectric effect, Am. J. Phys, 77, 87 (2009)

[18] E. F. Redish and E. Kuo, Language of Physics, Language of Math: Disciplinary Culture and Dynamic Epistemology, Sci. \& Educ. 24, 561-590 (2015)

[19] Photoelectric Effect Tutorial (for review)

[20] https://phet.colorado.edu/en/simulation/photoelectric.

[21] Lens Equation Activity (for review) 